\title{
Using analytic morphomics to describe body composition associated with post-kidney transplantation diabetes mellitus
}

\author{
David C. Cron ${ }^{1,2}$ (D) | Kelly A. Noon ${ }^{3}$ | Devan R. Cote ${ }^{3}$ | Michael N.Terjimanian ${ }^{1,2}$ (D) | \\ Joshua J. Augustine ${ }^{4}$ | Stewart C. Wang ${ }^{1,2}$ | Michael J. Englesbe ${ }^{1,2}$ | Kenneth J. Woodside ${ }^{1}$
}

${ }^{1}$ Department of Surgery, University of Michigan Medical School, Ann Arbor, MI, USA

${ }^{2}$ Morphomic Analysis Group, University of Michigan Medical School, Ann Arbor, MI, USA

${ }^{3}$ Department of Surgery, Case Western Reserve University and University Hospitals Case Medical Center, Cleveland, OH, USA

${ }^{4}$ Department of Internal Medicine, Case Western Reserve University and University Hospitals Case Medical Center, Cleveland, $\mathrm{OH}$, USA

\section{Correspondence}

Kenneth J. Woodside, MD, Section of

Transplant Surgery, Department of Surgery,

University of Michigan Health System, Ann Arbor, MI, USA.

Email: woodside@umich.edu

\begin{abstract}
Background: Better risk assessment tools are needed to predict post-transplantation diabetes mellitus (PTDM). Using analytic morphomic measurements from computed tomography (CT) scans, we aimed to identify specific measures of body composition associated with PTDM.

Methods: We retrospectively reviewed 99 non-diabetic kidney transplant recipients who received pre-transplant CT scans at a single institution between 1/2005 and $5 / 2014$. Analytic morphomic techniques were used to measure abdominal adiposity, abdominal size, and psoas muscle area and density, standardized by gender. We measured the associations of these morphomic factors with PTDM.

Results: One-year incidence of PTDM was $18 \%$. The morphomic factors significantly associated with PTDM included visceral fat area (OR=1.84 per standard deviation increase, $P=.020$ ), body depth (OR=1.79, $P=.035)$, and total body area (OR=1.67, $P=.049)$. Clinical factors significantly associated with PTDM included African American race (OR=3.01, $P=.044)$, hypertension $(O R=2.97, P=.041)$, and dialysis vintage $(O R=1.24$ per year on dialysis, $P=.048)$. Body mass index was not associated with PTDM (OR=1.05, $P=$.188). On multivariate modeling, visceral fat area was an independent predictor of PTDM (OR=1.91, $P=.035)$.

Conclusions: Analytic morphomics can identify pre-transplant measurements of body composition that are predictive of PTDM in kidney transplant recipients. Pretransplant imaging contains a wealth of underutilized data that may inform PTDM prevention strategies.
\end{abstract}

KEYWORDS

analytic morphomics, body composition, diabetes, kidney transplant, new-onset diabetes after transplant, obesity, post-transplant diabetes mellitus

\section{1 | INTRODUCTION}

Metabolic and cardiovascular complications are common following kidney transplantation and are a significant cause of post-transplant morbidity. Post-transplantation diabetes mellitus (PTDM, sometimes termed new-onset diabetes mellitus after transplantation) is common and affects $10 \%-15 \%$ of kidney transplant recipients. ${ }^{1-5}$ This complication is associated with worse post-transplant outcome, including diabetic complications, cardiovascular complications, and lower graft and patient survival. ${ }^{6-8}$ PTDM development is attributed in part to post-transplant immunosuppressant therapy. Namely, corticosteroids and tacrolimus can impair insulin sensitivity. ${ }^{3-5}$ However, patient-specific factors such as age, race, and hepatitis $\mathrm{C}$ viral infection are associated with the development of PTDM as well. ${ }^{4,5}$ Higher body 
mass index (BMI) is also associated with PTDM. ${ }^{5,9}$ Measurements of body composition represent important patient-specific modifiable risk factors; however, BMI fails to discriminate between fat and muscle, and thus provides an incomplete measure of body composition. More specific measurements of central adiposity, visceral and subcutaneous adiposity, and lean muscle mass may help identify PTDM risk factors and facilitate intervention.

Computed tomography (CT) scans can be used to delineate visceral adiposity from subcutaneous adiposity. Visceral adiposity is believed to be the more metabolically active fat and has been shown to be associated with insulin resistance. ${ }^{10}$ Analytic morphomics is a novel technology that uses semi-automated processing of CT scans to obtain granular measurements of body composition. ${ }^{11,12}$ This technology has been shown to predict surgical risk in myriad patient populations. ${ }^{11,13,14}$ Such CT-based methods have also been shown to identify markers of chronic disease such as cirrhosis and diabetes. ${ }^{15,16}$ Recently, our group has shown that analytic morphomics can identify specific body composition associated with PTDM following liver transplantation. ${ }^{17}$ The value of analytic morphomics in kidney transplantation is unclear. This technology may be able to address the need for better pre-kidney transplant assessment of PTDM risk.

To this end, we used analytic morphomic techniques to measure pre-transplant abdominal adiposity, body size, and trunk musculature, and we correlated these measurements with incidence of PTDM in kidney transplant recipients. We aimed to identify specific body composition risk factors for PTDM. We hypothesized that greater abdominal adiposity, larger abdominal size, and lower lean muscle mass are associated with higher incidence of PTDM.

\section{2 | METHODS}

\section{1 | Study population}

This study was reviewed and approved by the Institutional Review Boards of both the University Hospitals Case Medical Center (UHCMC, IRB\#07-13-31) and the University of Michigan Medical School (UMMS, IRB\#HUM00041441) and is adherent to the Declaration of Helsinki. This study was a retrospective analysis of patients who underwent kidney transplantation at UHCMC between January 2005 and May 2014 who had a CT scan in the year prior to transplant. At UHCMC, abdominopelvic or pelvic CT scans were ordered annually per clinical protocol for kidney transplant candidates who met any one of the following criteria: age $\geq 50$ years, diabetes, significant cardiovascular risk, or previous transplant. These scans were obtained to assess for iliac artery calcification and operative anastomotic targets. We also included CT scans ordered for other clinical reasons, as long as the scans were within 1 year prior to the transplant. In patients with multiple pre-transplant CT scans, we selected the scan closest to transplant. Protocol scans were non-contrast. We excluded patients who had clinically recognized pre-transplant diabetes or received a simultaneous pancreas or liver transplant.

Typical immediate peri-kidney transplant immunosuppression regimens at UHCMC included thymoglobulin, mycophenolate mofetil, tacrolimus, and 4 days of methylprednisolone. Tacrolimus was occasionally switched to cyclosporine in the setting of tacrolimus-related toxicity. Tacrolimus target levels were $8-12 \mathrm{ng} / \mathrm{mL}$ in first 90 days and $5-8 \mathrm{ng} / \mathrm{mL}$ thereafter. All patients typically received methylprednisolone for four days post-transplant. As part of a steroid avoidance protocol, steroids were then typically stopped or tapered, unless the patient was immunologically higher risk, had delayed graft function beyond the intravenous steroid course, or had pre-existing need for steroids, such as certain autoimmune diseases. For patients who were maintained on steroids post-transplant, prednisone maintenance dosing was typically $5 \mathrm{mg}$, and sometimes as low as $2.5 \mathrm{mg}$.

\section{2 | Clinical data}

Clinical data were retrospectively collected from the electronic medical record. Data were de-identified and coded and sent to UMMS to be merged with the de-identified and coded morphomic data. Clinical variables collected included age, sex, race, BMI, end-stage renal disease etiology (hypertension, autoimmune, or other), hepatitis $C$ viral infection, dialysis pre-transplant (including time on dialysis), prior kidney transplant, deceased donor, prednisone post-transplant, and tacrolimus or cyclosporine usage post-transplant.

\section{3 | Outcome}

\subsection{1 | Post-transplant diabetes mellitus}

All patients had at least 1 year of post-transplant data. Our outcome of interest was a binary measure indicating 1-year PTDM incidence. PTDM was retrospectively queried from the electronic medical record at UHCMC and was defined as peak fasting plasma glucose $\geq 126 \mathrm{mg} /$ $\mathrm{dL}$ or $\mathrm{HbA} 1 \mathrm{c} \geq 6.5 \%$. We insured that the PTDM persisted until 1-year post-transplant or patient death to exclude transient hyperglycemia.

\subsection{Analytic morphomics}

CT scans were de-identified and assigned unique identifiers before undergoing morphomic analysis by the University of Michigan Morphomic Analysis Group. Figure 1 shows the morphomic measures considered in this analysis: visceral fat area, subcutaneous fat area, subcutaneous fat anterior thickness, total body area, total body circumference, body depth (anterior-posterior axis), body width (lateral axis), total psoas area, and average psoas density. CT scans were processed using semi-automated methods programmed into MATLAB v. 2013a (MathWorks, Natick, MA, USA). Scans were processed by research students and full-time research associates, all of whom received extensive training. All scans were reviewed and approved by a single reviewer for quality control. The methodology for measurement of total psoas areas has been previously described. ${ }^{11}$ Briefly, scans were first indexed by vertebral level to enable precise measurements. All measurements for this study were taken at the L4 vertebral level. This level is most commonly used in morphomics studies, as the psoas muscle is most reliable measured at this level. Additionally, many of 


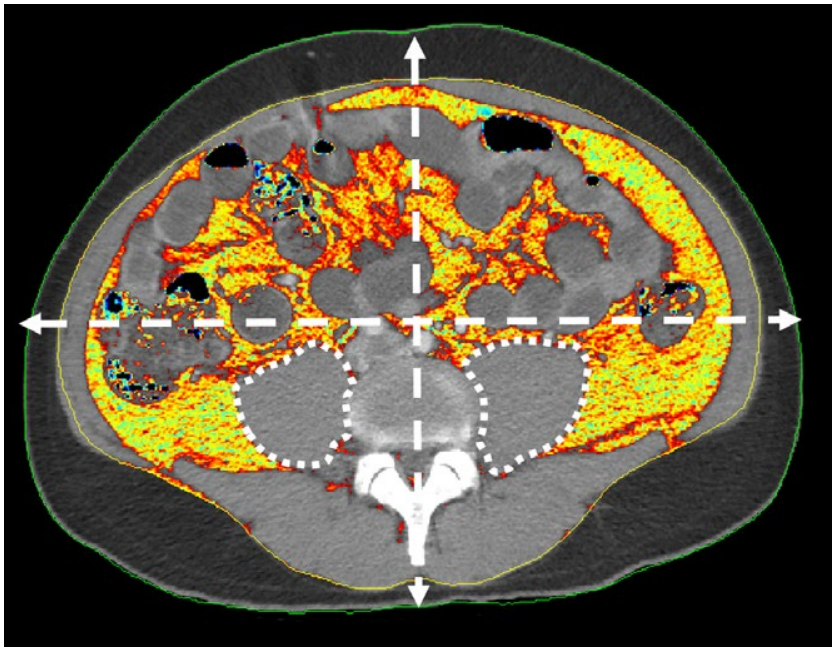

FIGURE 1 Morphomic measurements. Morphomic measurements are calculated from CT scans. The skin is outlined in green. The fascia is outlined in yellow. Within this fascial region, the visceral fat is portrayed by a density heat map. The psoas muscles are outlined by white dots. The vertical dashed line represents body depth (anteriorposterior axis), and the horizontal dashed line represents body width (lateral axis)

the scans in this study were pelvic (to assess iliac vessels) and thus did not contain levels superior to L4. Total psoas area was the sum of the left and right cross-sectional areas taken just inferior to the L4 vertebral level. Average psoas density was computed by measuring the Hounsfield units of each individual pixel and averaging these values across all pixels in bilateral psoas muscle cross-sectional slices. ${ }^{18}$ Larger values of Hounsfield units indicate more dense muscles (ie less fatty infiltration).

Fat areas and body size measurements were also measured at the L4 vertebral level. Methods for computing these measurements are described elsewhere. ${ }^{17,18}$ The fascial and skin envelopes were outlined. Measurements of body depth, width, circumference, and area were made relative to the skin envelope. The Hounsfield units of each pixel within the fascial envelope were calculated, and any pixels with density within the range of fat were deemed visceral fat. Similarly, Hounsfield units of pixels between the fascial and skin envelopes were used to determine subcutaneous fat.

\section{5 | Statistical analysis}

Continuous variables were summarized by mean and standard deviation (SD), or median and interquartile range (IQR) if skewed. Continuous variables were compared using a Student's $t$ test or a Wilcoxon rank-sum test, as appropriate. Pairwise correlations were assessed using Pearson's correlation coefficient $(r)$. Categorical variables were summarized by frequency tables and compared using Fisher's exact test. For regression analysis, morphomic variables were standardized by sex to account for variation in body size by sex. Univariate and multivariate logistic regression was used to assess the relationship of clinical and morphomic factors to PTDM, with results expressed as odds ratios (OR) with $95 \%$ confidence intervals $(\mathrm{Cl})$. For multivariate analysis, variables with a univariate $P \leq .05$ were included, and $\mathrm{BMI}$ was included regardless of statistical significance. Due to collinearity between different morphomic factors, we ran a separate multivariate model for each statistically significant morphomic predictor identified on univariate analysis. For graphical display, we dichotomized BMI, with $\mathrm{BMI} \geq 30$ considered high $\mathrm{BMI}$, which corresponded to $33 \%$ of the cohort. We also dichotomized visceral fat area, subcutaneous fat area, and total psoas area, with values in the upper tertile (gender-specific) for each measure considered high, and values in the lower and middle tertile considered low. We chose to dichotomize these measures because sample size and the binary outcome limited number of groups that could be used for categorization, and we were interested in comparing the upper extremes of body size. All significance tests were two-sided, with significance accepted at $P<.05$. All statistical analyses were performed using Stata v13.1 (StataCorp LP, College Station, TX, USA).

\section{3 | RESULTS}

A total of 99 patients met inclusion criteria. The median time from CT scan to transplant was 162 days (IQR 89-246). Table 1 shows demographic and clinical characteristics for the study cohort. The cohort was $65 \%$ male with a mean age of $52.9 \pm 13.5$, with $25.3 \%$ of the patients African American. The mean age was $52.9 \pm 13.5$ years, while the median dialysis vintage (years of dialysis pre-transplant) was 1.5 years (IQR 0-4.2). Thirteen patients (13\%) had a history of prior kidney transplant. In this cohort, $62 \%$ of patients continued prednisone post-transplant beyond the protocolled time point of post-operative

TABLE 1 Patient characteristics

\begin{tabular}{|lc|}
\hline Demographic variables & \\
\hline Male & 69 \\
\hline Age & $64(64.6 \%)$ \\
\hline African American & $52.9 \pm 13.5$ \\
\hline Body mass index & $25(25.3 \%)$ \\
\hline ESRD etiology & $27.9 \pm 6.4$ \\
\hline Hypertension & \\
\hline Autoimmune & $34(34.3 \%)$ \\
\hline Other etiology & $19(19.2 \%)$ \\
\hline Hepatitis C-positive & $54(54.5 \%)$ \\
\hline Dialysis pre-transplant & $7(7.1 \%)$ \\
\hline Dialysis vintage (years pre-transplant) & $74(74.7 \%)$ \\
\hline Prior kidney transplant & $1.5(0-4.2)$ \\
\hline Deceased donor & $13(13.1 \%)$ \\
\hline Prednisone continued post-transplant & $54(54.5 \%)$ \\
\hline Cyclosporine post-transplant & $61(61.6 \%)$ \\
\hline
\end{tabular}

Numbers represent $\mathrm{N}(\%)$ or mean $\pm \mathrm{SD}$; dialysis vintage is represented as median (interquartile range). 
day 4 , and $7 \%$ of patients received cyclosporine post-transplant. Morphomic measurements in the sample were all approximately normally distributed.

We assessed correlations between the gender-standardized morphomic measures and BMI. BMI was positively correlated with all morphomic measures of fat, muscle, and body size: visceral fat area $(r=.40)$, subcutaneous fat area $(r=.65)$, subcutaneous fat anterior thickness $(r=.64)$, total body area $(r=.68)$, total body circumference $(r=.68)$, body depth ( $r=.64)$, and body width ( $r=.64)$. BMI was weakly correlated with muscle measurements: total psoas area $(r=.24)$ and average psoas density $(r=.16$, although not significant, $P=.121)$. The correlation between visceral fat area and subcutaneous fat area was $r=.59$. Psoas area was weakly correlated with subcutaneous fat area $(r=.22)$ and was not significantly correlated with visceral fat area $(r=.05, P=.635)$.

The incidence of PTDM was $18 \%$ at 1-year post-transplant. Median time from transplant to PTDM diagnosis was 223 days (IQR 58-329). Of the 18 patients who developed PTDM, 13 gained weight by 1-year post-transplant, and average weight and $\mathrm{BMI}$ change of those 18 patients by 1 -year post-transplant was $6.9 \pm 12.5 \mathrm{~kg}$ and $2.3 \pm 4.3$, respectively. Regarding management of PTDM in these patients, five (27\%) were managed with insulin, six (33\%) with oral medication, and seven (39\%) were initially diet-controlled. HbA1c levels at 1-year post-transplant (within 3 months of this date) were available for nine (50\%) of the patients who developed PTDM and were on average 6.9 \pm 1.5 . Table 2 shows clinical factors associated with PTDM. Three factors were significantly associated with PTDM: African American race $(\mathrm{OR}=3.01,95 \% \mathrm{Cl}$ 1.03-8.80, $P=.044)$, hypertension $(\mathrm{OR}=2.97$, $95 \% \mathrm{Cl} 1.04-8.44, P=.041)$, and dialysis vintage ( $\mathrm{OR}=1.24$ per $y$ ear on dialysis, $95 \% \mathrm{Cl} 1.00-1.54, P=.048)$. BMI was not significantly associated with PTDM (OR=1.05, 95\% Cl 0.98-1.13, $P=.188)$. Other notable factors that were not significantly associated with PTDM included hepatitis $C$ viral infection $(P=.466)$ and continued prednisone use posttransplant $(P=.311)$.

The morphomic factors associated with PTDM are also shown in Table 2. The following variables were significantly associated with PTDM: visceral fat area (OR=1.84 per SD increase, 95\% Cl 1.10-3.09, $P=.020)$, body depth (OR=1.79 per SD increase, 95\% Cl 1.04-3.09, $P=.035)$, and total body area (OR=1.67 per SD increase, $95 \% \mathrm{Cl} 1.00$ 2.77, $P=.049$ ). Morphomic factors that trended toward significance included subcutaneous fat area $(P=.086)$, subcutaneous fat anterior thickness $(P=.065)$, and total body circumference $(P=.075)$. Of note, neither total psoas area nor average psoas density were associated with PTDM ( $P=.113$ and $P=.700$, respectively).

The morphomic measurements compared between patients with and without incident PTDM are shown in Table 3. Without accounting for gender, patients who developed PTDM had 35\% greater visceral fat area $(P=.047)$. There was a trend toward greater body depth in patients who developed PTDM (9\% greater, $P=.057)$. Among patients with PTDM, there was no difference in morphomic measurements in insulin-controlled vs oral medication or diet-controlled patients. Figure 2 shows unadjusted rates of PTDM incidence by high and low visceral fat, subcutaneous fat, total psoas area, and BMI. Rates of PTDM were 2.6-fold higher in patients with high visceral fat area
TABLE 2 Factors associated with post-transplant diabetes mellitus

\begin{tabular}{|c|c|c|c|}
\hline & OR & 95\% C.I. & $P$-value \\
\hline \multicolumn{4}{|l|}{ Clinical variables } \\
\hline Male & 0.63 & $(0.22-1.76)$ & .375 \\
\hline Age & 1.01 & $(0.97-1.05)$ & .615 \\
\hline African American & 3.01 & $(1.03-8.80)$ & .044 \\
\hline Body mass index & 1.05 & (0.98-1.13) & .188 \\
\hline \multicolumn{4}{|l|}{ ESRD etiology } \\
\hline Hypertension & 2.97 & $(1.04-8.44)$ & .041 \\
\hline Autoimmune & 0.47 & $(0.10-2.25)$ & .345 \\
\hline Other etiology & 0.61 & $(0.22-1.70)$ & .344 \\
\hline Dialysis pre-transplant & 3.17 & (0.68-14.91) & .144 \\
\hline $\begin{array}{l}\text { Dialysis vintage (years } \\
\text { pre-transplant) }\end{array}$ & 1.24 & $(1.00-1.54)$ & .048 \\
\hline Hepatitis C-positive & 1.90 & (0.34-10.68) & .466 \\
\hline $\begin{array}{l}\text { Prednisone } \\
\text { post-transplant }\end{array}$ & 1.79 & $(0.58-5.49)$ & .311 \\
\hline \multicolumn{4}{|l|}{ Morphomic variables } \\
\hline Visceral fat area & 1.84 & $(1.10-3.09)$ & .020 \\
\hline Subcutaneous fat area & 1.56 & $(0.94-2.58)$ & .086 \\
\hline $\begin{array}{l}\text { Subcutaneous fat } \\
\text { anterior thickness }\end{array}$ & 1.61 & $(0.97-2.66)$ & .065 \\
\hline Total body area & 1.67 & $(1.00-2.77)$ & .049 \\
\hline $\begin{array}{l}\text { Total body } \\
\text { circumference }\end{array}$ & 1.60 & $(0.95-2.69)$ & .075 \\
\hline Body depth & 1.79 & $(1.04-3.09)$ & .035 \\
\hline Body width & 1.25 & $(0.76-2.08)$ & .380 \\
\hline Total psoas area & 1.51 & $(0.91-2.51)$ & .113 \\
\hline Average psoas density & 1.11 & $(0.65-1.89)$ & .700 \\
\hline
\end{tabular}

C.I.=confidence interval.

Clinical and morphomic variables underwent univariate logistic regression. Morphomic variables were standardized by gender and coded in standard deviation units. Bold values indicate $P<.05$.

compared to low (31\% vs $12 \%, P=.027)$. PTDM rates were not significantly different in patients with high vs low subcutaneous fat area ( $19 \%$ vs $18 \%, P=1.000)$, psoas area ( $22 \%$ vs $16 \%, P=.581)$, or BMI $(21 \%$ vs $17 \%, P=.590$ ).

For multivariate analysis, one model was run for each of the three morphomic factors identified as statistically significant on univariate analysis (visceral fat area, total body area, and body depth). Due to collinearity between morphomic factors, we chose a priori to analyze each of these three morphomic factors in three separate multivariate models. Each model adjusted for BMI, hypertension, African American race, and dialysis vintage. The results of these models are shown in Table 4. Visceral fat area was an independent predictor of PTDM (OR=1.91 per standard deviation increase, 95\% Cl 1.05-3.48, $P=.035)$. Neither total body area (OR=1.76, 95\% $\mathrm{Cl} 0.87-3.56, P=.115)$ nor body depth $(\mathrm{OR}=1.84,95 \% \mathrm{Cl} 0.92-3.67, P=.086)$ were statistically significant predictors of PTDM on multivariate analysis. No other 
TABLE 3 Morphomic measures by post-transplant diabetes mellitus status
FIGURE 2 Post-transplantation diabetes mellitus (PTDM) incidence by fat, muscle, and body mass index (BMI). This graph shows 1-y PTDM incidence by high/ low muscle, fat, and BMI. The high group is designated as the upper gender-specific tertile of fat or muscle area, or $\mathrm{BMI} \geq 30$. The low group is designated as the lower or middle gender-specific tertile of fat or muscle area, or $\mathrm{BMI}<30\left({ }^{*} P<.05\right)$

\begin{tabular}{|lccc|}
\hline Morphomic component & $\begin{array}{l}\text { No post-transplantation } \\
\text { diabetes mellitus (PTDM) }\end{array}$ & PTDM & P \\
\hline Visceral fat $\left(\mathrm{mm}^{2}\right)$ & $14799 \pm 9103$ & $19996 \pm 9620$ & .047 \\
\hline Subcutaneous fat $\left(\mathrm{mm}^{2}\right)$ & $23500 \pm 12640$ & $29927 \pm 17992$ & .165 \\
\hline $\begin{array}{l}\text { Total body area }\left(\mathrm{mm}^{2}\right) \\
\text { Total body circumference }\end{array}$ & $71753 \pm 20713$ & $82247 \pm 25905$ & .122 \\
\hline (mm) & $983 \pm 143$ & $1049 \pm 174$ & .149 \\
\hline Body depth $(\mathrm{mm})$ & $250 \pm 40$ & $272 \pm 43$ & .057 \\
\hline Body width (mm) & $353 \pm 53$ & $363 \pm 64$ & .506 \\
\hline $\begin{array}{l}\text { Total psoas area }\left(\mathrm{mm}^{2}\right) \\
\text { Average psoas density } \\
\text { (Hounsfield units) }\end{array}$ & $2294 \pm 751$ & $2351 \pm 566$ & .721 \\
\hline
\end{tabular}

Numbers represent mean \pm SD for all measures. Bold values indicate $P<.05$.

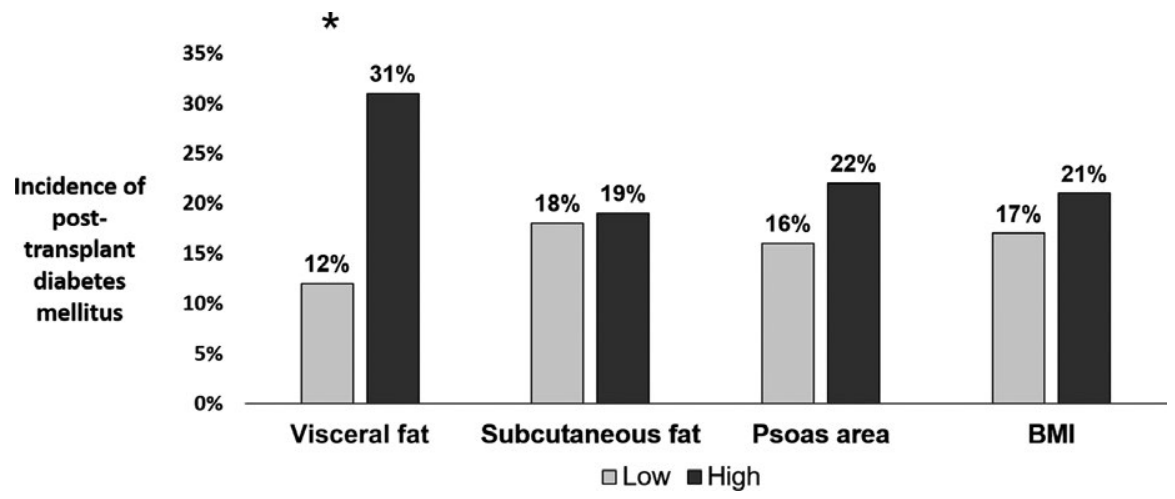

TAB LE 4 Multivariate logistic regression

\begin{tabular}{|c|c|c|c|c|c|c|c|c|c|}
\hline & \multicolumn{3}{|l|}{ Model 1} & \multicolumn{3}{|l|}{ Model 2} & \multicolumn{3}{|l|}{ Model 3} \\
\hline Visceral fat area & 1.91 & (1.05-3.48) & .035 & & & & & & \\
\hline Total body area & & & & 1.76 & $(0.87-3.56)$ & .115 & & & \\
\hline Body depth & & & & & & & 1.84 & $(0.92-3.67)$ & .086 \\
\hline Body mass index & 1.02 & $(0.93-1.11)$ & .692 & 0.99 & $(0.89-1.11)$ & .921 & 1.00 & $(0.90-1.10)$ & .973 \\
\hline African American & 3.23 & $(0.82-12.63)$ & .092 & 2.26 & $(0.65-7.94)$ & .202 & 2.17 & $(0.62-7.53)$ & .224 \\
\hline $\begin{array}{l}\text { Dialysis vintage (years } \\
\text { pre-transplant) }\end{array}$ & 1.14 & $(0.90-1.45)$ & .287 & 1.15 & $(0.90-1.46)$ & .261 & 1.16 & $(0.91-1.47)$ & .235 \\
\hline
\end{tabular}

C.I.=confidence interval.

Clinical and morphomic variables underwent multivariate logistic regression. A separate multivariate model was run for each of visceral fat area, total body area, and body depth. Morphomic variables were standardized by gender and coded in standard deviation units. Bold values indicate $P<.05$.

clinical factors were statistically significantly associated with PTDM on multivariate analysis.

\section{4 | DISCUSSION}

We explored the utility of analytic morphomics in identifying body composition associated with PTDM in kidney transplant recipients.
The prevalence of PTDM in this study was $18 \%$ at 1 -year posttransplant. We identified multiple specific measures of body fat and body size that were significantly associated with PTDM, including visceral fat area, total body area, and body depth. In a multivariate model adjusting for clinical factors, visceral fat area alone remained independently and significantly associated with PTDM. Interestingly, BMI was not significantly associated with PTDM in this cohort. The correlations between the morphomic measures and 
BMI were variable; measures of body size (total body area, body circumference, and body depth) were most strongly correlated with BMI. Pre-transplant imaging contains a wealth of patient-specific health markers that can be used for risk assessment. These findings suggest that analytic morphomics can be used to identify granular measurements of body composition that are predictive of PTDM.

Although BMI is well established as a predictor of PTDM, its flaws are known. BMI does not account for regional fat distribution or amount of lean muscle mass-factors that are known to determine metabolic risk. Central adiposity is known to be metabolically more active and higher risk compared to peripheral adiposity. ${ }^{19-21}$ Specifically, abdominal visceral fat is believed to be more strongly associated with insulin resistance and diabetes compared to abdominal subcutaneous fat. ${ }^{10,21}$ Additionally, lower lean muscle mass has been associated with insulin resistance. ${ }^{22}$ Analytic morphomic analysis of abdominal CT scans can discriminate regional fat distribution and lean muscle mass, and this technology may provide a useful risk assessment tool in kidney transplant recipients

This study highlights the potential utility of pre-transplant CT scans for PTDM risk assessment. Our previous work in liver transplantation identified subcutaneous fat thickness as the only significant predictor of PTDM. ${ }^{17}$ This current study suggests that analytic morphomics may have more utility in predicting PTDM in kidney transplant recipients than liver recipients. Other studies have shown specific body composition correlates of PTDM, although few have used pre-transplant measures to predict incidence of PTDM. One cross-sectional study found higher visceral fat in PTDM patients (measured post-transplant) compared to controls. ${ }^{23}$ Another study found that PTDM patients had greater waist circumference compared to controls (also measured post-transplant). ${ }^{24}$ Interestingly, BMI is known to be a predictor of PTDM $^{5,9}$ but was not a significant predictor in our sample. This is not to suggest that BMI does not predict PTDM, as sample size may be inadequate to show this difference, even as some morphomic predictors have adequate sample size to show such a difference. Rather, as morphomic measures are more specific than the composite measurement of $\mathrm{BMI}$, our results suggest that morphomic measurements may be more predictive than BMI. Additionally, other previously established PTDM risk factors such as steroid use and hepatitis $C$ were not predictive of PTDM in this study, and this finding likely reflects the study cohort characteristics (eg, steroid avoidance protocol) and sample size limitations. Our study adds to this literature by showing that specific pre-transplant measures of fat area and body size are predictive of PTDM incidence.

CT scans are frequently ordered prior to kidney transplantation, yet the full potential of these scans is not being utilized. Beyond operative planning, CT scans can be used to identify high-risk surgical candidates, ${ }^{11,13}$ and we suggest they also can be used to predict metabolic outcomes such as PTDM. In the context of PTDM, stronger methods of risk assessment are needed to identify high-risk patients who can benefit from intervention. For example, assessing specific fat and muscle measurements in kidney transplant candidates may facilitate development of nutrition and exercise interventions that are tailored to the patient's specific needs. We have had success with similar pre-operative optimization programs in general surgery patients at our institution. ${ }^{25,26}$ Further, immunosuppression regimens can be tailored according to the patient's PTDM risk, but such clinical decisions require precise risk assessment to optimally balance metabolic and immunologic risk. Given the results of this pilot study, we hypothesize that analytic morphomics may be able to improve risk assessment by incorporating specific measures of body composition into predictive models. Analytic morphomics has been shown to improve the discriminative ability of predictive models in other contexts. ${ }^{12,15}$ As a follow-up to this initial work, we plan to expand our database to multiple institutions. With a larger sample size, our future work will aim to develop robust multivariate models incorporating morphomics for prediction of PTDM.

This study has limitations. This is a single-center study, and thus the results may not be generalizable across other centers- which may partially account for differences from previously published studies from other centers. As a retrospective study, we were limited in the data we were able to collect. In particular, family history of diabetes was not reliably recorded, and consistent intervals between patients for tacrolimus dosages and levels were not available, as the patients were not in a prospective protocol. As tacrolimus has been associated with PTDM, this lack of reliable data limits ascribing PTDM etiology. Prospective collection of additional pre-transplant metabolic data would better enable us to account for baseline metabolic risk. Our sample size was a limitation, and future studies with larger sample size would enable robust multivariate predictive modeling. However, the results of this pilot study will guide our future work as we aim to identify the independent predictive utility of analytic morphomics in the context of PTDM. Lastly, in our sample, CT scans were ordered for operative planning in patients who were older than 50 , had significant cardiovascular risk, or had other clinical indications warranting imaging. This analyzed population is biased toward such older and sicker patients; these findings may not be generalizable to all populations.

In conclusion, we demonstrated that analytic morphomics can identify specific pre-transplant measurements of body composition that are predictive of PTDM incidence in kidney transplant recipients. The results of this study may serve as a proof-of-concept, suggesting utility of analytic morphomics for predicting PTDM risk in kidney transplant recipients. Morphomic analysis may add to a robust assessment of PTDM risk. Such PTDM risk assessment may help mitigate PTDM by not only targeting pre-transplant risk factors, but also through tailoring immunosuppressive and other post-transplant medications, lifestyle interventions, and nutritional support. Additional work is ongoing to expand upon these results and further explore the predictive ability and clinical utility of this novel technology.

\section{AUTHORS' CONTRIBUTIONS}

David C. Cron: Conception and design, acquisition of data, and analysis and interpretation of data; Kelly A. Noon: Conception and design, and acquisition of data; Devan R. Cote: Conception and design, and acquisition of data; Michael N. Terjimanian: Conception and design, and interpretation of data; Joshua J. Augustine: Conception 
and design, acquisition of data, and interpretation of data; Stewart C. Wang: Conception and design, acquisition of data, and interpretation of data; Michael J. Englesbe: conception and design, acquisition of data, and interpretation of data; Kenneth J. Woodside: Conception and design, acquisition of data, and interpretation of data; All authors aided in drafting or critically revising the article and approved the final manuscript.

\section{POTENTIAL CONFLICT OF INTEREST}

SCW and MJE are equity stakeholders in Prehab Technologies, LLC.

\section{REFERENCES}

1. Sharif A, Hecking M, de Vries AP, et al. Proceedings from an international consensus meeting on posttransplantation diabetes mellitus: recommendations and future directions. Am J Transplant. 2014;14:1992-2000.

2. Porrini E, Moreno JM, Osuna A, et al. Prediabetes in patients receiving tacrolimus in the first year after kidney transplantation: a prospective and multicenter study. Transplantation. 2008;85:1133-1138.

3. Luan FL, Steffick DE, Ojo AO. New-onset diabetes mellitus in kidney transplant recipients discharged on steroid-free immunosuppression. Transplantation. 2011;91:334-341.

4. Montori VM, Basu A, Erwin PJ, Velosa JA, Gabriel SE, Kudva YC Posttransplantation diabetes: a systematic review of the literature. Diabetes Care. 2002;25:583-592.

5. Shah T, Kasravi A, Huang E, et al. Risk factors for development of newonset diabetes mellitus after kidney transplantation. Transplantation. 2006;82:1673-1676.

6. Burroughs TE, Swindle J, Takemoto S, et al. Diabetic complications associated with new-onset diabetes mellitus in renal transplant recipients. Transplantation. 2007;83:1027-1034.

7. Kasiske BL, Snyder JJ, Gilbertson D, Matas AJ. Diabetes mellitus after kidney transplantation in the United States. Am J Transplant. 2003;3:178-185.

8. Lentine KL, Brennan DC, Schnitzler MA. Incidence and predictors of myocardial infarction after kidney transplantation. J Am Soc Nephrol. 2005; 16:496-506

9. Lafranca JA, JN IJ, Betjes MG, Dor FJ. Body mass index and outcome in renal transplant recipients: a systematic review and meta-analysis. BMC Med. 2015;13:111.

10. Hayashi T, Boyko EJ, McNeely MJ, Leonetti DL, Kahn SE, Fujimoto WY. Visceral adiposity, not abdominal subcutaneous fat area, is associated with an increase in future insulin resistance in Japanese Americans. Diabetes. 2008;57:1269-1275.

11. Englesbe MJ, Lee JS, He K, et al. Analytic morphomics, core muscle size, and surgical outcomes. Ann Surg. 2012;256:255-261.

12. Zhang P, Parenteau C, Wang L, et al. Prediction of thoracic injury severity in frontal impacts by selected anatomical morphomic variables through model-averaged logistic regression approach. Accid Anal Prev. 2013;60:172-180

13. Englesbe MJ, Patel SP, He K, et al. Sarcopenia and mortality after liver transplantation. J Am Coll Surg. 2010;211:271-278.

14. Lee JS, He K, Harbaugh CM, et al. Frailty, core muscle size, and mortality in patients undergoing open abdominal aortic aneurysm repair. J Vasc Surg. 2011;53:912-917.

15. Krishnamurthy $\mathrm{V}$, Zhang P, Ethiraj $\mathrm{S}$, et al. Use of analytic morphomics of liver, spleen, and body composition to identify patients at risk for cirrhosis. Clin Gastroenterol Hepatol. 2015;13:360-368.e365

16. Smith SR, Lovejoy JC, Greenway F, et al. Contributions of total body fat, abdominal subcutaneous adipose tissue compartments, and visceral adipose tissue to the metabolic complications of obesity. Metabolism. 2001;50:425-435.

17. Vaughn V, Cron DC, Terjimanian MN, et al. Analytic morphomics identifies predictors of new-onset diabetes after liver transplantation. Clin Transplant. 2015;29:458-464.

18. Zhang P, Peterson M, Su GL, Wang SC. Visceral adiposity is negatively associated with bone density and muscle attenuation. Am J Clin Nutr. 2015;101:337-343.

19. Kannel WB, Cupples LA, Ramaswami R, Stokes J 3rd, Kreger BE, Higgins M. Regional obesity and risk of cardiovascular disease; the Framingham Study. J Clin Epidemiol. 1991;44:183-190.

20. Misra A, Vikram NK. Clinical and pathophysiological consequences of abdominal adiposity and abdominal adipose tissue depots. Nutrition. 2003;19:457-466.

21. Bray GA, Jablonski KA, Fujimoto WY, et al. Relation of central adiposity and body mass index to the development of diabetes in the Diabetes Prevention Program. Am J Clin Nutr. 2008;87:1212-1218.

22. Rattarasarn C, Leelawattana R, Soonthornpun S. Contribution of skeletal muscle mass on sex differences in 2-hour plasma glucose levels after oral glucose load in Thai subjects with normal glucose tolerance. Metabolism. 2010;59:172-176.

23. von During ME, Jenssen T, Bollerslev J, et al. Visceral fat is better related to impaired glucose metabolism than body mass index after kidney transplantation. Transpl Int. 2015;28:1162-1171.

24. Dedinska I, Laca L, Miklusica J, et al. Waist circumference as an independent risk factor for NODAT. Ann Transplant. 2015;20:154-159.

25. Friedman J, Lussiez A, Sullivan J, Wang S, Englesbe M. Implications of sarcopenia in major surgery. Nutr Clin Pract. 2015;30:175-179.

26. Englesbe MJ, Lussiez AD, Friedman JF, Sullivan JA, Wang SC. Starting a surgical home. Ann Surg. 2015;262:901-903.

How to cite this article: Cron DC, Noon KA, Cote DR, et al. Using analytic morphomics to describe body composition associated with post-kidney transplantation diabetes mellitus. Clin Transplant. 2017;31:e13040.

https://doi.org/10.1111/ctr.13040 